\title{
afmToolkit: an R Package for Automated AFM Force-Distance Curves Analysis
}

\author{
by Rafael Benítez, Vicente J. Bolós and José-Luis Toca-Herrera
}

\begin{abstract}
Atomic force microscopy (AFM) is widely used to measure molecular and colloidal interactions as well as mechanical properties of biomaterials. In this paper the afmToolkit $\mathrm{R}$ package is introduced. This package allows the user to automatically batch process AFM force-distance and force-time curves. afm Toolkit capabilities range from importing ASCII files and preprocessing the curves (contact point detection, baseline correction...) for finding relevant physical information, such as Young's modulus, adhesion energies and exponential decay for force relaxation and creep experiments. This package also contains plotting, summary and feature extraction functions. The package also comes with several data sets so the user can test the aforementioned features with ease. The package afmToolkit eases the basic processing of large amount of AFM F- $d / t$ curves at once. It is also flexible enough to easily incorporate new functions as they are needed and can be seen as a programming infrastructure for further algorithm development.
\end{abstract}

\section{Introduction}

In the last thirty years, atomic force microscopy (AFM) has become a necessary surface analytical tool for life and materials scientists (Müller and Dufrêne, 2008; Kainz et al., 2014). AFM offers the possibility to investigate molecular topographies and dynamical processes at (sub) nanometer scale as a function of time (Hansma et al., 1996; Ortega-Vinuesa et al., 1998; Kuznetsov et al., 2010; Lopez et al., 2010).

In addition, the AFM is a mechanical machine being able to measure forces between molecules, particles or surfaces (Hinterdorfer et al., 1996; Butt et al., 2005; Borkovec et al., 2012). It can also be used to measure mechanical properties of biomaterials, either indenting or stretching them (Rief, 1997; Marszalek et al., 1999; Alcaraz et al., 2003; Best et al., 2003; Kasas and Dietler, 2008; Garcia-Manyes and Sanz, 2010; Benitez and Toca-Herrera, 2014; Melzak and Toca-Herrera, 2015).

One problem that the researcher faces while performing force-distance (F-d) curves is the handling and the interpretation of the amount of data. For example, for determining the unbinding force between two molecules, hundreds of curves might be needed. Therefore, adaptive and flexible software routines are necessary to export, analyze and organize the measured data before starting the physical data interpretation. Several authors have addressed this type of problem before, proposing algorithms to F-d curve signals (Benítez et al., 2013; Kasas et al., 2000; Andreopoulos and Labudde, 2011; Crick and Yin, 2007; Lin et al., 2007a). However, these works focused mostly on contact point detection, adhesion energy quantification, or unfolding events. Here we present a set of functions bundled in a package of R statistical software that offer a compact analysis of the whole F-d curve.

\section{Force - distance curve parts}

In force-distance experiments, an AFM-tip or a colloidal probe (see Ducker et al. (1991)) is extended towards and retracted from the sample at speeds that may vary between a few $\mathrm{nm} / \mathrm{s}$ and dozens of $\mu \mathrm{m} / \mathrm{s}$. While performing the F-d experiment, the deflection of the cantilever is quantified as a function of the displacement of the piezo-scanner (Moreno-Flores and Toca-Herrera, 2013). Thus, the force sensed by the cantilever is calculated by multiplying its deflection by its spring constant (Hooke's law), which must be evaluated in every experiment. A general F-d curve (or force-time curve) can be divided in three parts: move towards the sample, contact with the sample and separation from the sample. Every part of the curve contains valuable and different information about the sample of study. The first one delivers information about repulsive, attractive or structural forces between the tip/colloidal probe and the sample (e.g. electrostatic, van der Waals, hydration, entropic, etc.). The second part of the curve corresponds to the so-called contact regime, that is, when the cantilever is touching (compressing) the sample. This part of the curve provides information about the mechanical properties of the sample (i.e. Young's modulus, relaxation time and viscosity of cells or hydrogels). Finally, the separation curve contains information about adhesion or rupture forces (i.e. ligand-receptor interactions), the existence of tethers, and possible molecular unfolding events (i.e. mechanical unfolding of polymers).

However, the operating way of the piezo-electric does not follow this segmentation. The piezoelectric distinguishes three movements: approach, pause and retract (see Figure 1). In this way, the F-d raw data that can be acquired from the AFM is structured in these three parts. Indeed, it does not 
know where the contact point is located (zero distance between tip and sample).
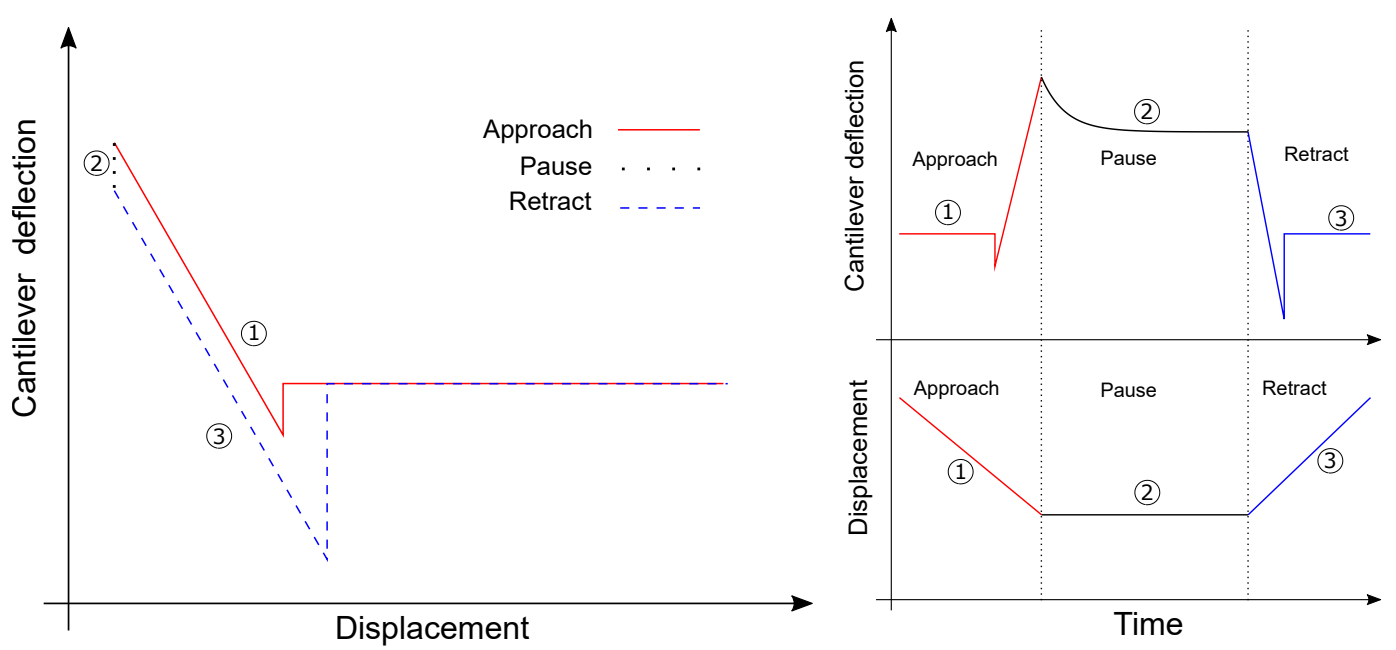

Figure 1: F-d curve. Left: Schematic representation of a typical Force-distance curve. Right (above and below): Schematic representation of a typical Force-time at constant height.

Therefore, it is necessary to extract all above mentioned physically relevant information from the F-d raw data as it is given by the device.

\section{The afm Toolkit package}

In this article we introduce the afmToolkit package whose aim is to automate certain operations and calculations that are normally done routinely on the F-d curves.

Package afm Toolkit is available in CRAN and can be installed via the command 'install . package'. Nevertheless, the development version of the package of afmToolkit is also stored in the github platform (github.com) and it can be installed directly from the R console using the 'install_github' function from package devtools (Wickham and Chang, 2016).

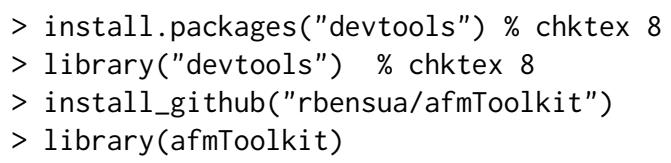

The package depends on the ggplot2 package (Wickham, 2016a) and uses functions from the non-standard packages minpack.lm, (Elzhov et al., 2015), gridExtra (Auguie, 2016), scales (Wickham, 2016b) and dplyr (Wickham and Francois, 2015).

\section{The "afmdata" class}

The basic data structure for AFM F-d curves analysis with the afmToolkit package is the "afmdata" class. It is an S3 class consisting on a list having at least the fields data and params (see Figure 2).

The field data is a data frame containing the data itself. The columns are $\mathrm{Z}$, for the distance, Force and segment, being the later a factor with levels approach, pause and/or retract, denoting which part of the force - distance curve each data belongs to. In some cases also a Time column could be present.

Eventually the number of columns of the data data frame may be increased, as different analysis are performed. For example, once the baseline correction is done, a new column ForceCorrected is added.

The params field is a list containing different parameters gathering information about the experiment (e.g. the cantilever spring constant, the ID of the curve, etc.).

As further analyses are performed, the results are added as new fields to the "afmdata" list. Therefore, the "afmdata" list will eventually contain, in a single data structure, the whole F-d curve relevant information. 


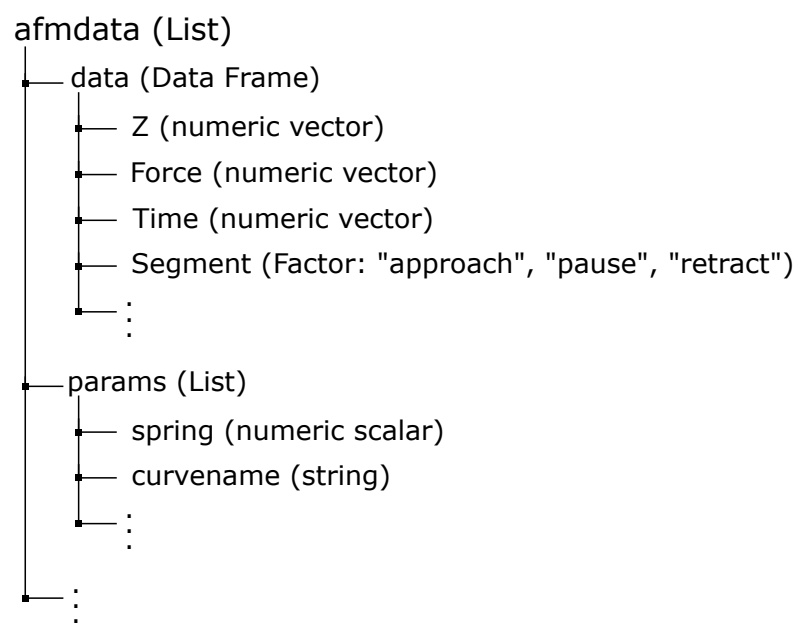

Figure 2: "afmdata" class. Basic "afmdata" class list description.

\section{Importing data}

Although the "afmdata" class definition function is flexible enough to create an "afmdata" structure from a data frame directly, it is usually more convenient, in order to speed up the work flow, to import the data from a file obtained directly from the AFM device used.

At the moment, there are only available two functions for importing F-d curves: afmReadJPK and afmReadVeeco, which import NanoWizard JPK and Veeco (Bruker) data files, respectively, provided they had previously been exported as ASCII files.

Importing data from JPK ${ }^{\mathrm{TM}}$ ASCII files: In the first case, a full AFM experiment is stored in a single text file in which the different segments of the experiment (approach, contact or pause, and retract) are separated by a header (see Figure 3-Left).
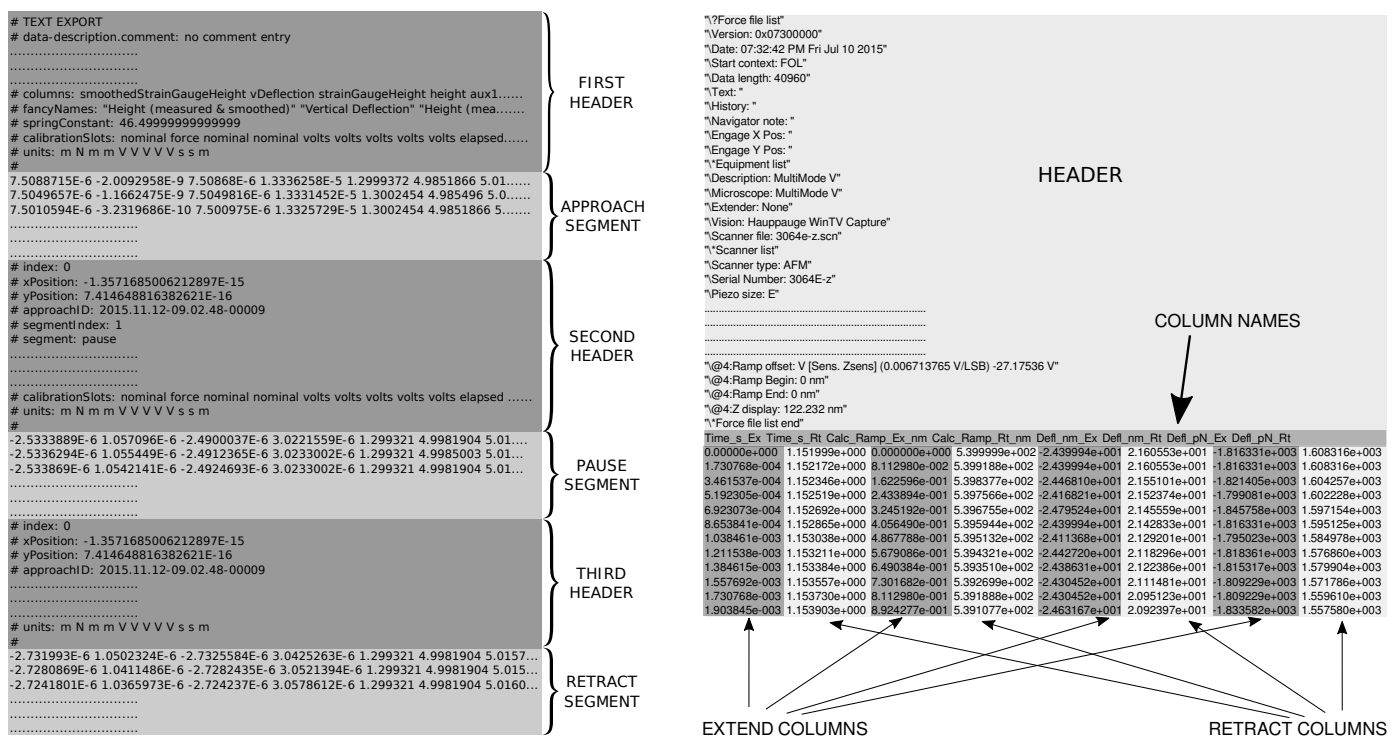

Figure 3: ASCII Files. Left: structure of a JPK ASCII file with three headers. Right: Structure of a Veeco (Bruker) ASCII file.

The function finds out how many headers the file contains (up to three headers), which columns contain the relevant information: distance, force and time (if available), and it also looks for the spring constant in the header and stores it in the params field. The ID (curvename) of the experiment will be the name of the ASCII file (by default). 
The afmToolkit has two JPK text example files: 'force-save-JPK-2h.txt' and 'force-save-JPK-3h.txt', with two and three segments, respectively. Let's see an example.

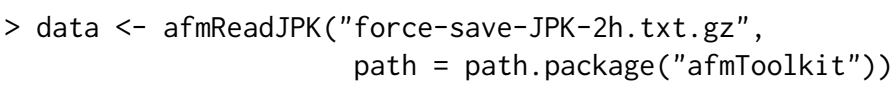

Once it is loaded we can check the structure of the new "afmdata" variable created.

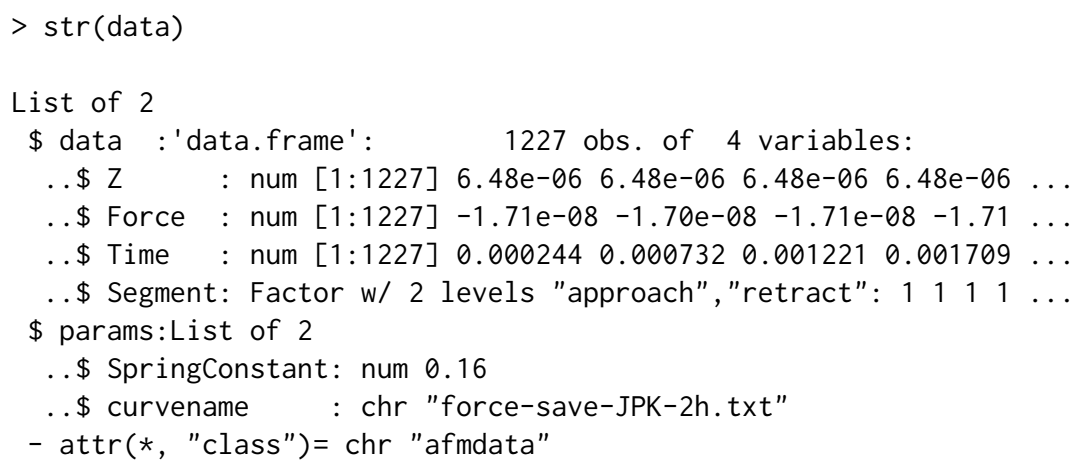

From the output we can see that there are two fields in the "afmdata" structure: the data field, and the params field.

We can easily plot the whole experiment using the available plotting S3-method plot.afmdata, which makes use of the ggplot2 package.

$>\operatorname{plot}($ data $)$

The result is depicted in Figure 4.

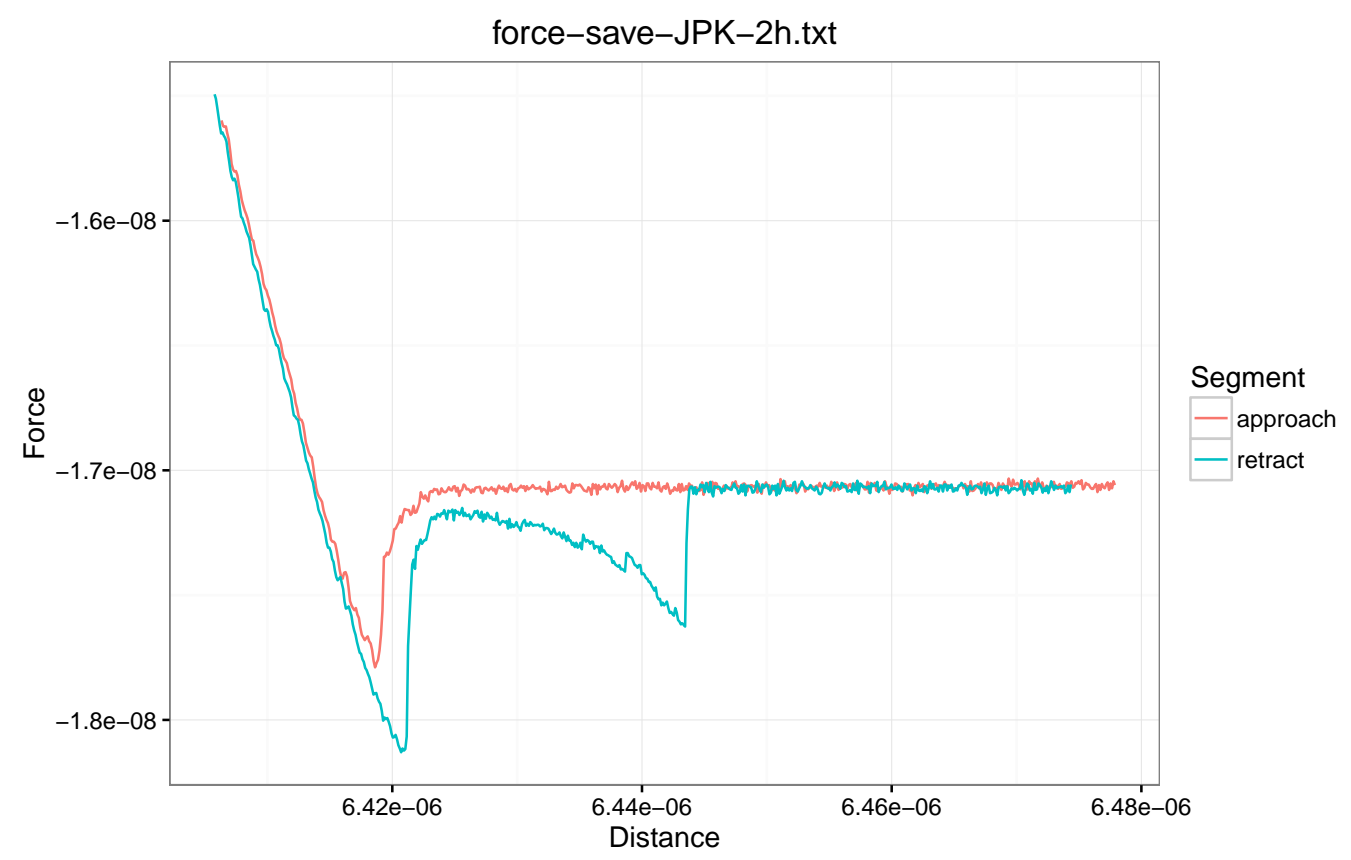

Figure 4: plot. afmdata function. Two segment JPK F-d curve plotted with the plot . afmdata function. JPK refers to the type of AFM. In this case the cantilever moves and the sample is at rest. For the Multimode (Bruker) the cantilever is at rest and the sample moves.

Note that the afmReadJPK function automatically determines the number of segments from the number of headers present in the ASCII file. However it does not find out from the headers which segment is each one of them. That is, if afmReadJPK finds only one header, it will assume that the file contains only an approach segment. If two headers are detected, it will be assumed that the first one is the approach and the second is the retract segment. Finally, if three headers are found, they will be assigned to the approach, pause and retract, respectively. At this moment no more than three segments per file are supported. 
Importing data from Veeco ${ }^{\mathrm{TM}}$ ASCII files: A Veeco ASCII file is, in comparison, simpler than the JPK file. It contains a single header with the details of the parameters of the AFM experiment and the data from each part of the experiment (approach or extend, and retract) are stored on separated columns (see Figure 3-right).

The afmReadVeeco function reads the data file and creates an "afmdata" structure separating the different parts of the experiment obtained from their corresponding columns in the file.

The syntax for importing a Veeco file is very similar to the one of the JPK file.

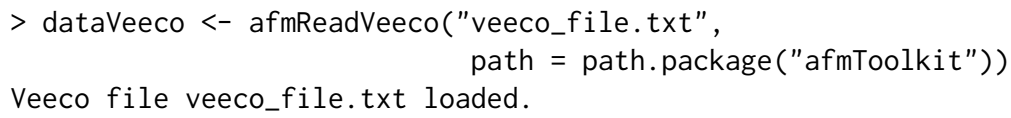

\section{Contact point and detach point determination}

Contact point: The first, and probably the most important step in the AFM F-d curve analysis is the determination of the contact point. We define such point as the location in the approach segment of the F-d curve where the deflection of the cantilever is, for the first time, significantly higher than the baseline average slope. It should be noted that we are using here the term "contact point" in a broad sense, since there is no necessity for a real contact between the tip and the sample to take place, but we are rather considering the contact point as the point at which the interactions between the sample and the tip start to appear. Such interactions could be caused by an actual contact between tip and sample, or they could be the response to a repulsive force or even a "jump to contact" attractive interaction.

Lots of different approaches to the determination of the contact point have already been made (Lin et al., 2007a,b; Rudoy et al., 2010; Benítez et al., 2013; Gavara, 2016). Function afmContactPoint estimates the contact point using the algorithm described in Benítez et al. (2013). This method computes, from the F-d signal, a new $\delta$ signal which is, roughly speaking, the lagged difference between two values of the slopes of the best lines fitted by a local linear regression on a rolling window of some predetermined width. High absolute values of $\delta$ are related to abrupt changes in either the original F-d curve or its slope. From the $\delta$ signal, the contact point is obtained using two given thresholds which are multiples of the $\delta$ signal noise (i.e. standard deviation) in the first part of the curve (i.e. non-contact part). See Benítez et al. (2013) for specific details on the algorithm.

For example, for the two segments example shown above, we could take the following parameters:

- width: Width of the window, given in number of points, in which the local regression is performed. We shall set width $=20$.

- mul1: Value of the first multiplier used to determine the first threshold. It should be small enough to detect the contact point accurately (even zero is a possible value). In this case we will set mul1 $=1$.

- mul2: Value of the second multiplier used to determine the second threshold. Its value should be large enough to distinguish the contact point from the regular noise of the signal but no so large that the contact point remains undetected. We will set this value as mul2 $=10$.

The rest of the parameter will remain in their default values. Then the following command will detect the contact point and plot it together with the approach segment of the F-d curve.

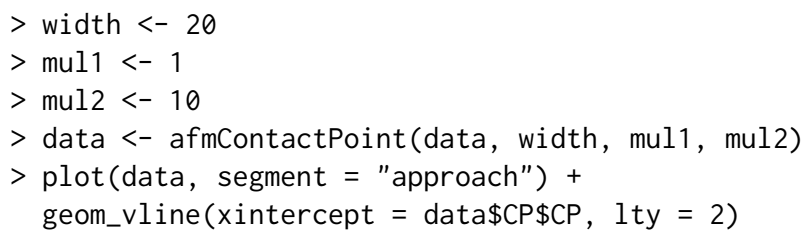

Figure 5-Left shows the approach segment of the curve together with the contact point estimation (dashed vertical line). Note that the contact point is the first point in the approach curve (from right to left) in which the curve starts to deviate from the baseline. When there is an attraction force (like in the example) this contact point is overestimated and, in order to be sure that we are starting the contact regime in which the tip is indeed contacting the sample, we should calculate the zero force point (see below).

Detach point: The detach point is the point in the retract segment where the tip finally leaves the sample. It is computed exactly in the same way that the contact point, but the computations are performed to the retract part and the curve is traced backwards. The input parameters of function afmDetachPoint are the same than for function afmContactPoint. Let us continue with our example: 

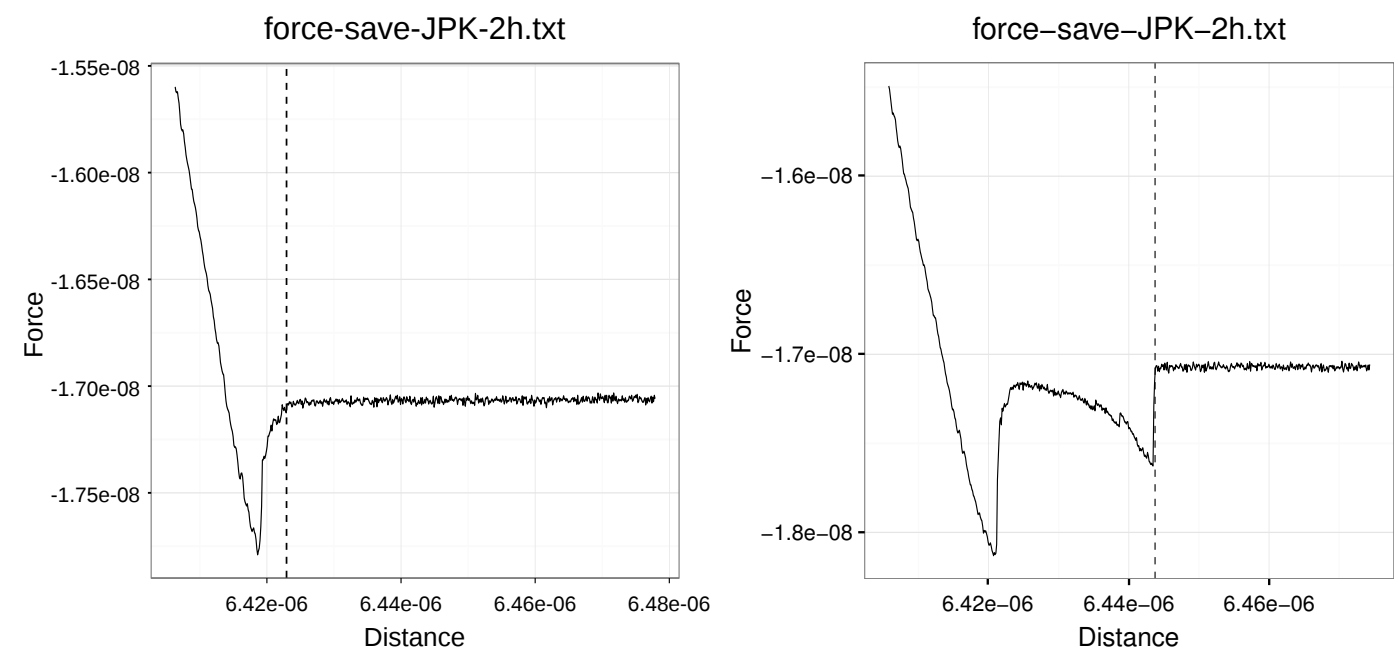

Figure 5: Contact point/Detach point detection. Left: F-d approach curve (solid line) and the estimation of the contact point (dashed line) with the afmContactPoint function. Right: Detach point (dashed line) estimated on the retract segment (solid line) with afmDetachPoint.

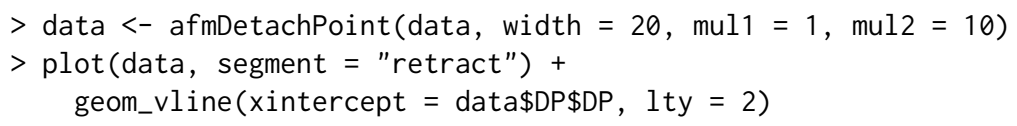

The estimation of the detach point can be seen in Figure 5-Right.

\section{Baseline correction}

Once the contact and detach points are found, the baseline calibration can be carried out. Theoretically, when the tip is far from the sample, the deflection of the cantilever and therefore the measured force should be zero. Nevertheless, in most cases there is an offset, or even a drift that keeps this part of the curve away from the zero value. In order to fix this behaviour, a baseline correction is done. Such correction is usually done manually, by selecting the part of the curve which we know to be away from the sample and then substract to the whole F-d curve, the least squares fitted line to such selected segment of the curve.

Since we already determined the contact and detach points, we know exactly when the tip is away from the sample. Function afmBaselineCorrection will perform this calibration automatically. This function will add a new column called ForceCorrected to the data data frame field of the "afmdata" class.

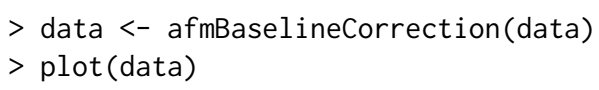

Once the baseline correction is done, futher analyses will only use the Corrected Force column of the data field. For example, the 'plot (data)' command will plot the "afmdata" F-d curve with the calibration already done, as can be observed in Figure 6 where it can be seen that when the tip is away from the sample, the force is actually zero.

\section{Zero force point}

As we mentioned above, function afmContactPoint finds the first point in the approach segment for which the slope of the curve is significantly different from the baseline. This usually coincides with what would be considered the contact point by "eye inspection". Nevertheless, when there is an attraction force prior to the contact regime, the point found with function afmContactPoint is an overestimation of the real contact point since it does not distinguish between attraction and repulsions. Therefore, a new function, afmZeroPointSlope, can be used to find the point after the minimum which is the intersection between the curve and the baseline (zero force line).

With the following commands we can obtain the zero force point and the slope of the F-d curve after that point. The results will be added to the data structure in new field named Slope. We finally plot the curve and both in Figure 7, the contact point (dashed red line) and the zero force point (blue dotted line). 


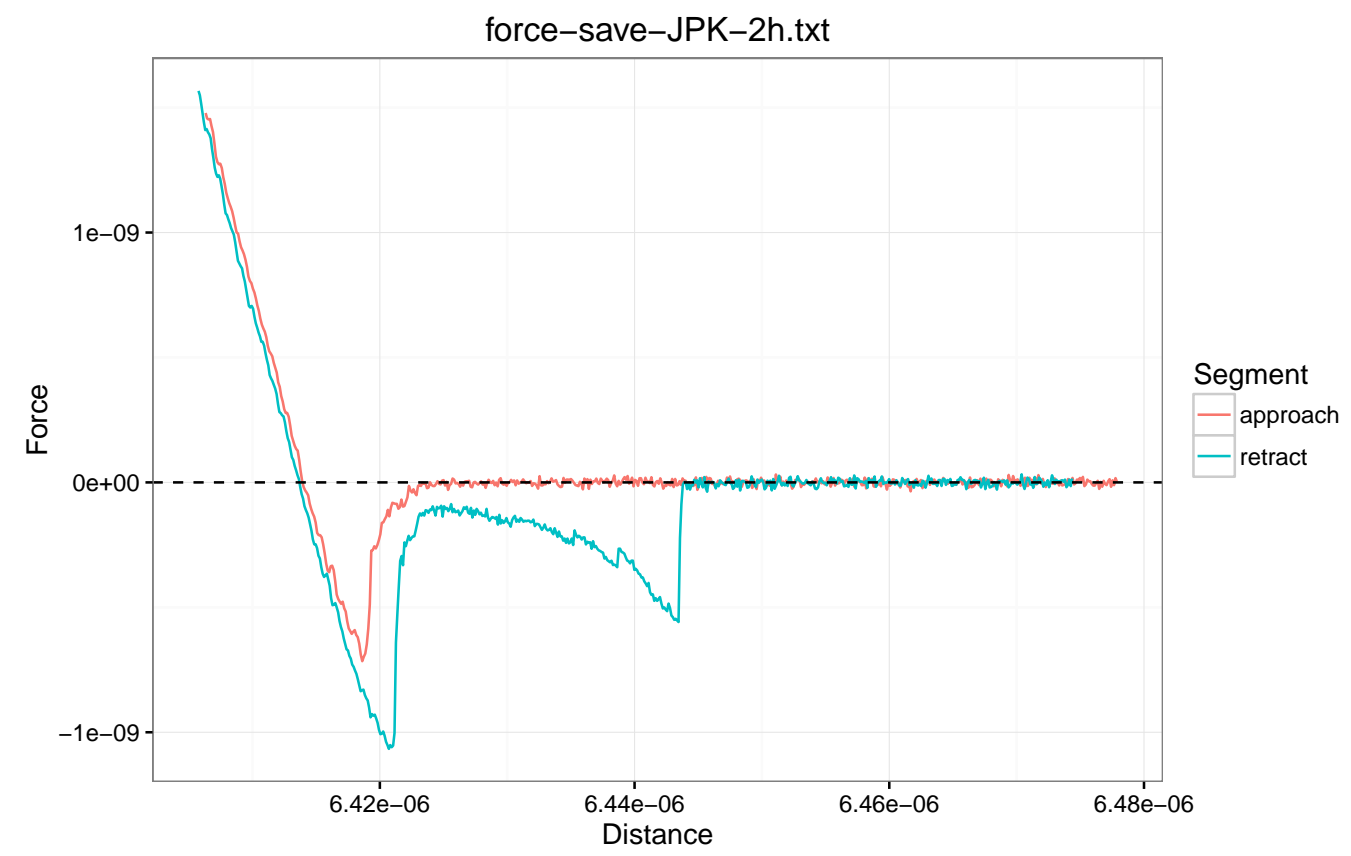

Figure 6: Base line correction.

F-d curve with the baseline correction done with afmBaselineCorrection function.

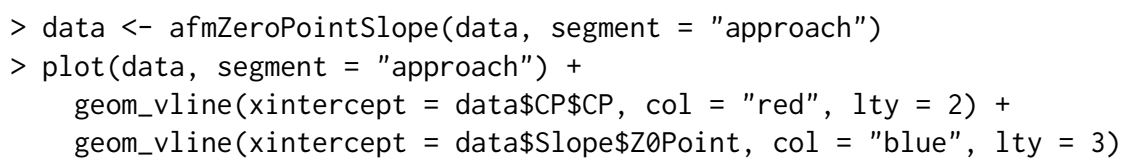

\section{Young's modulus estimation}

One of the most important parameters for determining the mechanical properties of a sample is the Young's modulus. Obtaining the Young's modulus from an AFM F-d curve is not straightforward and depends on several factors. Namely, the spring constant of the cantilever, the contact area, which largely depends on the tip's geometry and the Poisson ratio, which depends on the compressibility of the sample. The typical AFM tip geometries are: spherical (colloidal probes), pyramidal and conical.

Function afmYoungModulus computes the Young's modulus of the sample from the approach segment fo the force-distance curve. Before it is called, be aware that the spring constant should be available in the params field of the "afmdata" structure and both, the baseline correction and the zero force point should have been obtained.

Currently, only the two most used geometries are available for this function: the four-sided pyramidal tip and the paraboloid tip. The former uses the classical Snedon formulae:

$$
F=\frac{E}{1-v^{2}} \frac{\tan \alpha}{\sqrt{2}} \delta^{2},
$$

being $E$ the Young's modulus, $v$ the Poisson ratio, $\alpha$ the pyramid face angle and $\delta$ the indentation of the tip into the sample. Parameters $v$ and $\alpha$ should be provided ( $v=0.5$ is the default value) and first we will need to determine the tip's indentation.

The latter uses the Hertz model given by

$$
F=\frac{4 \sqrt{R}}{3} \frac{E}{1-v^{2}} \delta^{3 / 2} .
$$

The indentation can be obtained substracting to the piezo displacement, $Z$, the zero force point, $Z_{0}$ and the deflection of the cantilever,

$$
\delta=Z-Z_{0}-\frac{F}{\kappa}
$$

where $\kappa$ is the cantilever's spring constant. Function afmIndentation computes the indentation and adds it as a new column to the data field of the "afmdata" structure. Once the indentation is calculated, 


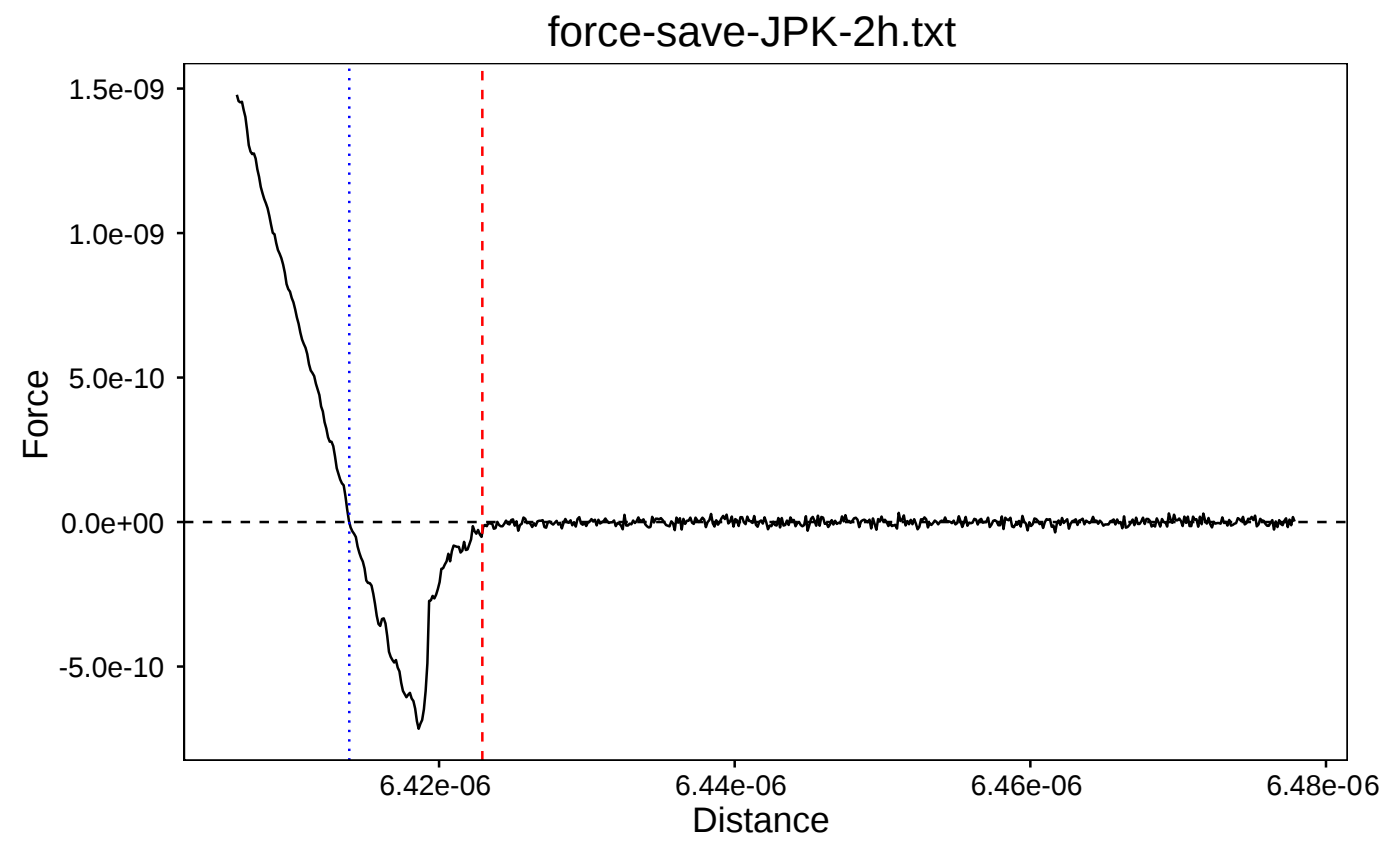

Figure 7: Zero-force point. Contact point (red dashed line) and zero force point (blue dotted line) obtained with afmContactPoint and afmZeroPointSlope, respectively.

function afmYoungModulus computes the Young's modulus by fitting a straight line to $F$ vs $\delta^{2}$. From the slope of the line fitted and (1), E can be obtained and it is added to the params field of the "afmdata" variable.

For the example data, using a pyramidal tip with $\alpha=22$ deg considering an incompressible sample $(v=0.5)$, we have

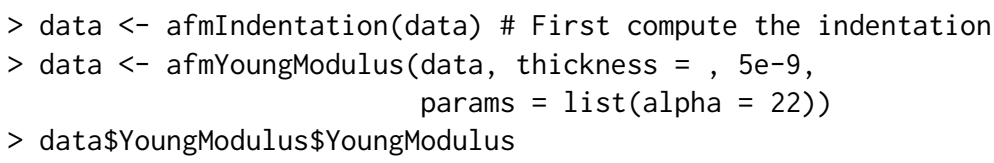

\section{[1] 59730377}

We have, therefore, obtained a Young's modulus of $E=59.73 \mathrm{MPa}$.

\section{Exponential decay fit}

Another important type of experiment used to obtain viscoelastic mechanical properties of the sample is the Force relaxation - Creep experiment. In these experiments, after reaching the sample, the tip remains in contact for a predetermined elapsed time. In the Force relaxation experiment, the height of the AFM's piezo is held constant, and in presence of a viscoelastic material, an exponential decay in the force should be observed. On the other hand, in a Creep experiment, the force remains constant and, as a consequence, it is the height of the tip what shows an exponential decay behaviour.

The presence of the exponential decays in force and/or in height, can be explained in terms of the classical linear viscoelastic theory, where combinations of Maxwell and Voight elements (springs and dahspots) are used. However they will not be discussed here and we refer the reader to Riande et al. (1999) for a general discussion of linear viscoleasticity and Moreno-Flores et al. (2010a,b) for more specific models used in nanoindentation AFM experiments in cell mechanics problems.

Plainly speaking, each different viscoelastic material in the sample is characterized by a different relaxation time. Thus, the response Force vs Time or $\mathrm{Z}$ vs Time could be represented by a Prony series of the form (following the notation of Moreno-Flores et al. (2010a)):

$$
F(t)=a_{0}+\sum_{k=1}^{n} a_{k} e^{t / \tau_{k}}
$$


for the Force relaxation experiment, and

$$
Z(t)=c_{0}+\sum_{k=1}^{n} c_{k} e^{x_{k} t}
$$

for the creep experiment.

The afmToolkit can determine the parameters of the above mentioned Prony series for both types of experiments by fitting the sum of exponential to the data via a nonlinear least squares LevenbergMardquart algorithm provided by the minpack.lm package (Elzhov et al., 2015). Presently, only either one or two exponentials in the Prony series can be considered. This can be explained because in typical AFM experiments, there is usually either an homogenenous material or, for cell mechanics problems, at least two materials - cell membrane and cell cytoskeleton - are considered.

An important issue when performing nonlinear least square fits is the election of the initial values for the parameters. Often there is an extremely high sensitivity to such values, so it is critical to make good initial guesses. For a general single exponential decay function $y(t)=a_{0}+a_{1} \exp \left(-t / \tau_{1}\right)$, we find that $a_{0}$ is the horizontal asymptote, $a_{0}+a_{1}$ is the value at $t=0$ and $\tau_{1}$ should be of the same order of magnitude of the total time. In case there is a two-exponential decay function $y(t)=$ $a_{0}+a_{1} \exp \left(-t / \tau_{1}\right)+a_{2} \exp \left(-t / \tau_{2}\right), a_{0}$ is again the horizontal asymptote, but now $a_{0}+a_{1}+a_{2}$ is the initial value $y(0)$, so a good initial guess could be setting both parameters $a_{1}$ and $a_{2}$ with the same values (i.e. $\left.a_{1}=a_{2}=\left(y(0)-a_{0}\right) / 2\right)$. For the initial values of the decay times (or frequencies), a usually good guess is to set one of them, say $\tau_{1}$, of the same order of magnitude as the total time, and then set the second one an order of magnitude smaller (i.e. $\tau_{2}=\tau_{1} / 10$ ).

Let us see an example. We will need a data file with three segments: approach, contact and retract.

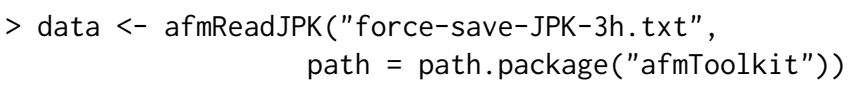

Once the data is loaded, we will proceed with the contact and detach point determination, baseline correction and the zero force point estimation.

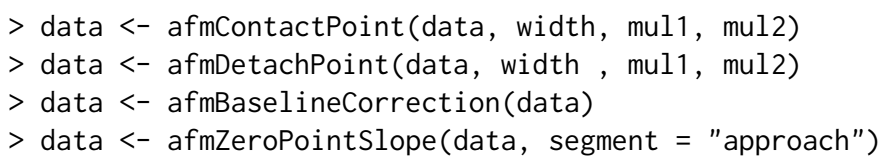

We may now plot the Force vs Time curve in the contact segment:

$>\operatorname{plot}($ data, segment $=$ "pause", vs $=$ "Time")

In Figure 8 it is shown the Force vs. Time curve together with the values of the magnitudes that will be used to make the initial guesses for the parameters.

Taking into account the values depicted in Figure 8, we shall see two options for the starting values of the fit parameters, one for the single exponential fit and other for the two exponentials fit.

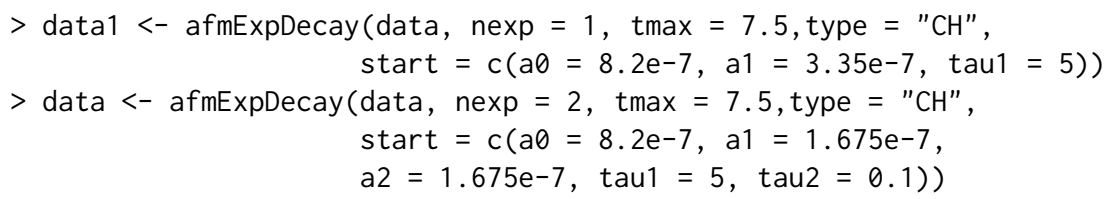

The output of this function is another afmdata class variable with an extra Expfit field, which is a list with two fields: expdecayModel and expdecayFit, being the former the nls class structure resulting from the nlsLM function from package minpack.lm, and the latter a numerical vector containing the exponentially decaying forces estimated by the fit.

Therefore, the standard summary function can be used to extract the relevant information of the fits:

$>$ summary (data1\$ExpFit\$expdecayModel)

$>$ summary (data\$ExpFit\$expdecayModel)

The fits are shown in Figure 9. It is seen that in this case, the double exponential clearly beats the single exponential fit, but in order to be sure we can check the goodness of fit data. From the fit summaries shown in Table 1 we may assert that the double exponential fit performs a better prediction than the single exponential fit - the Residual standard error is an order of magnitude smaller - while keeping all the parameters statistically significant, i.e. the standard error of all coefficient are at least one order of magnitude smaller than the value estimated. 


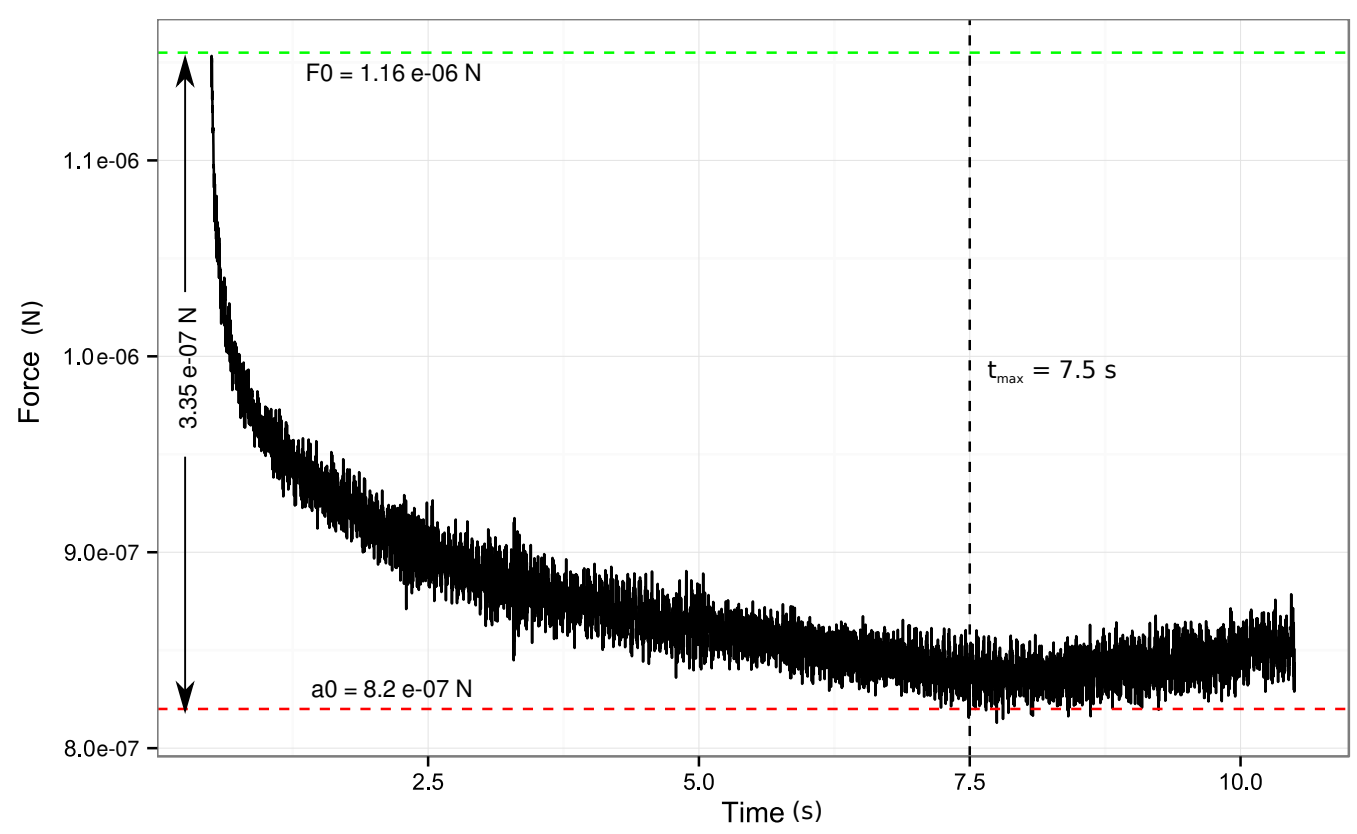

Figure 8: Force exponential decay. Force vs. Time plot in the contact segment. A clear exponential decay is observed. The important magnitudes are indicated in the plot.

\section{Adhesion energy}

When the cantilever retracts from the sample several events can take place, depending on the type of experiment. One of the most important effects that may occur is the adhesion phenomena. In a force spectroscopy experiment, the adhesion event is usually reflected in the F-d curve as an hysteresis loop, in which, as a result of the presence of non-conservative forces, the retract curve is below the approach curve.

The adhesion energy can be estimated as the area between the retract force-distance curve and the $\mathrm{Z}$-axis from the zero-force point - in which it is considered that the tip starts to detach from the sample - to the point at which there is a jump-from-contact event.

Sometimes, after this jump-from-contact event, some other important phenomena, like tether formation, can take place before the tip fully detaches from the sample and the F-d curve finally enters in the off-contact region.

We can compute these energies by means of the afmAdhesionEnergy function. This function uses an algorithm similar to the contact point estimation method implemented in the afmContactPoint and afmDetachPoint functions. It has as inputs the width of the rolling window in which a best line fit is computed and a multiplier mul that will be used to determine the jumps in the F-d curve that determine the different adhesion events.

Following the example of the three segment F-d curve, we could easily find the adhesion energies with the commands

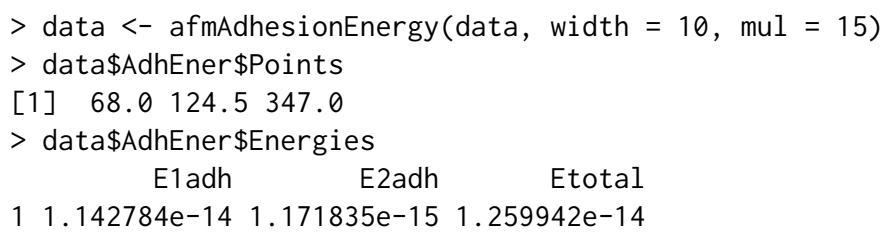

The afmAdhesionEnergy function appends to the afmdata class data input another field named AdhEner, which is a list with two fields: Points and Energies. The Points field is a vector of length 3 containing the indices of the F-d curve where the three events take place: the zero-force point (left end), the jump-from contact event (middle) and the full-detach event point (right end). The Energies field is also a vector of three components containing the adhesion energy, E1 adh, computed from the zero-force point to the jump-from-contact point, the remaining energy, E2adh, computed from the jump-from-contact point to the full-detach event point, and the total energy Etotal which is the sum of these two. Therefore, in this example, the total adhesion energy is around $1.2610^{-14} \mathrm{~J}$. 


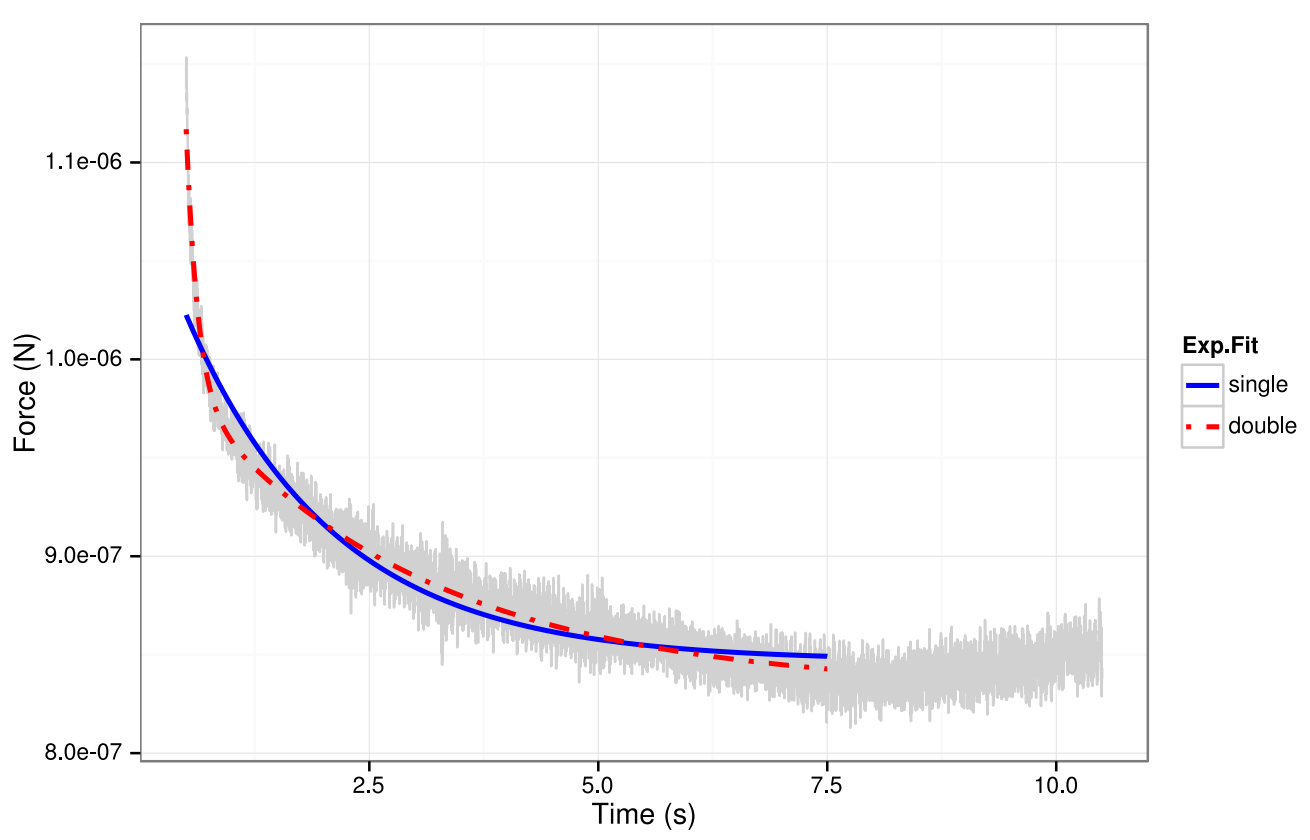

Figure 9: Exponential decay fits. Single and double exponential fits.

\section{Summarizing an "afmdata" class}

After all these analyses have been done to an F-d curve stored in an "afmdata" class, function summary can display the most relevant information about the curve in both, numerical and visual ways.

In order to illustrate a full example, we will first compute the Young's modulus of the three segments F-d curve example.

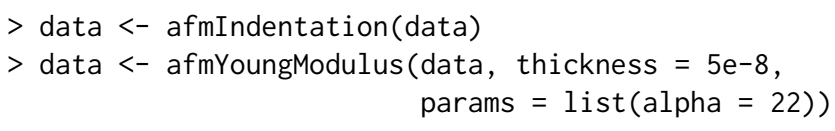

Now that all analyses are performed, let us see how the summary function shows us all relevant information.

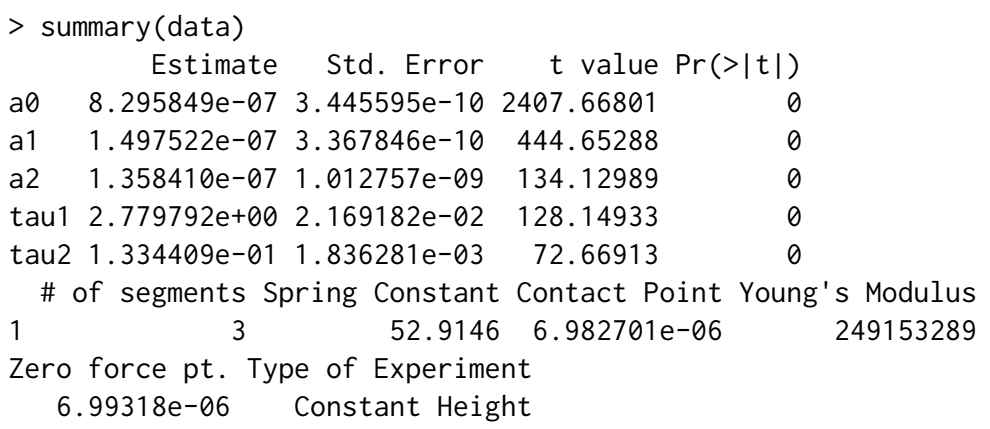

The graphical information provided is depicted in Figure 10. Thus, from the plots one can easily know the number of segments of the F-d curve, the goodness of the exponential decay fit, the value of the Young's modulus obtained with an Hertz's contact model and a pyramidal tip, among other useful information.

\section{A sample $\mathrm{R}$ afm Toolkit session for batch processing}

Up to now, we have shown the capabilities of the afmToolkit package for dealing with one F-d curve. However, the usual workflow in AFM force spectroscopy experiments is to repeat the measures a number of times that can be very large (even hundreds of repetitions). Therefore it is absolutely necessary algorithms and methods allowing us to batch-process all the curves at once. 
Table 1: Comparison of the single and double exponential fits.

Single exponential

\begin{tabular}{rrrcr}
\hline & Estimate & Std. Error & $\mathrm{t}$ value & $\operatorname{Pr}(>|\mathrm{t}|)$ \\
\hline$a_{0}$ & $8.5 \mathrm{e}-07$ & $2.3 \mathrm{e}-10$ & $3.6 \mathrm{e}+03$ & 0 \\
$a_{1}$ & $1.8 \mathrm{e}-07$ & $4.3 \mathrm{e}-10$ & $4.1 \mathrm{e}+02$ & 0 \\
$\tau_{1}$ & 1.6 & 0.0095 & $1.7 \mathrm{e}+02$ & 0 \\
\hline \multicolumn{4}{l}{ Residual standard error: $1.232 \mathrm{e}-08$ on 14333 d.o.f. }
\end{tabular}

Double exponential

\begin{tabular}{rrrrr}
\hline & Estimate & Std. Error & $\mathrm{t}$ value & $\operatorname{Pr}(>|\mathrm{t}|)$ \\
\hline$a_{0}$ & $8.3 \mathrm{e}-07$ & $3.6 \mathrm{e}-10$ & $2.3 \mathrm{e}+03$ & 0 \\
$a_{1}$ & $1.5 \mathrm{e}-07$ & $3.5 \mathrm{e}-10$ & $4.3 \mathrm{e}+02$ & 0 \\
$a_{2}$ & $1.4 \mathrm{e}-07$ & $1 \mathrm{e}-09$ & $1.3 \mathrm{e}+02$ & 0 \\
$\tau_{1}$ & 2.6 & 0.021 & $1.2 \mathrm{e}+02$ & 0 \\
$\tau_{2}$ & 0.13 & 0.0018 & 71 & 0 \\
\hline
\end{tabular}

The afmToolokit package can deal with a set of F-d curves stored as "afmdata" class variables and bundled together in one special data class called "afmexperiment".

An "afmexperiment" data class is a list of "afmdata" variables. Almost every function in the afmToolkit package first checks for the input data class. If the input is of "afmdata" class, it performs the analysis for one curve, but if it is an "afmexperiment" class variable, it loops for every "afmdata" curve in the list, executing the function to each individual F-d curve.

Next, we will show as an example, how would it be to deal with several F-d curves at once. First we will use the afmReadJPKFolder function in order to read all JPK files contained in some folder.

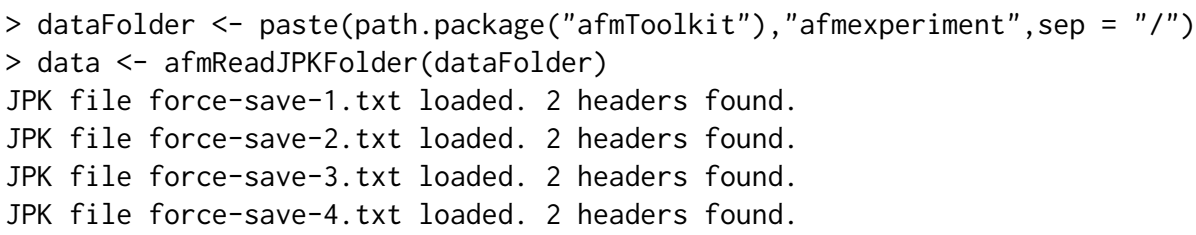

This function will create the "afmexperiment" data variable:

$>\operatorname{class}($ data)

[1] "afmexperiment"

As an illustrative example we can make use of the batchExperiment data set available in afmToolkit. This data set consists on an "afmexperiment" data class containing 14 F-d curves in "afmdata" format.

$>$ data(batchExperiment)

The 14 curves correspond to two different experiments: the first one is a sample covered with Polyallylamine hydrochloride ("PAH") (6 curves) while the second one is covered with Chitosan ("CHI") (8 curves). Such factor is specified in the params field of the "afmdata" structure. For example:

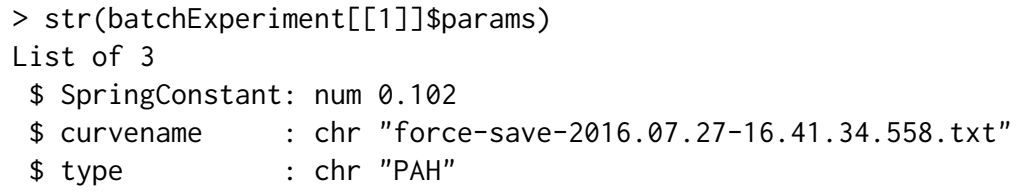

Now, the standard analysis procedure would be:

1. Preprocessing the curves: Contact and detach points detection, Baseline correction, Zero force point determination and indentation calculus.

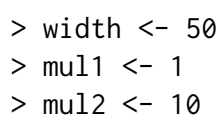



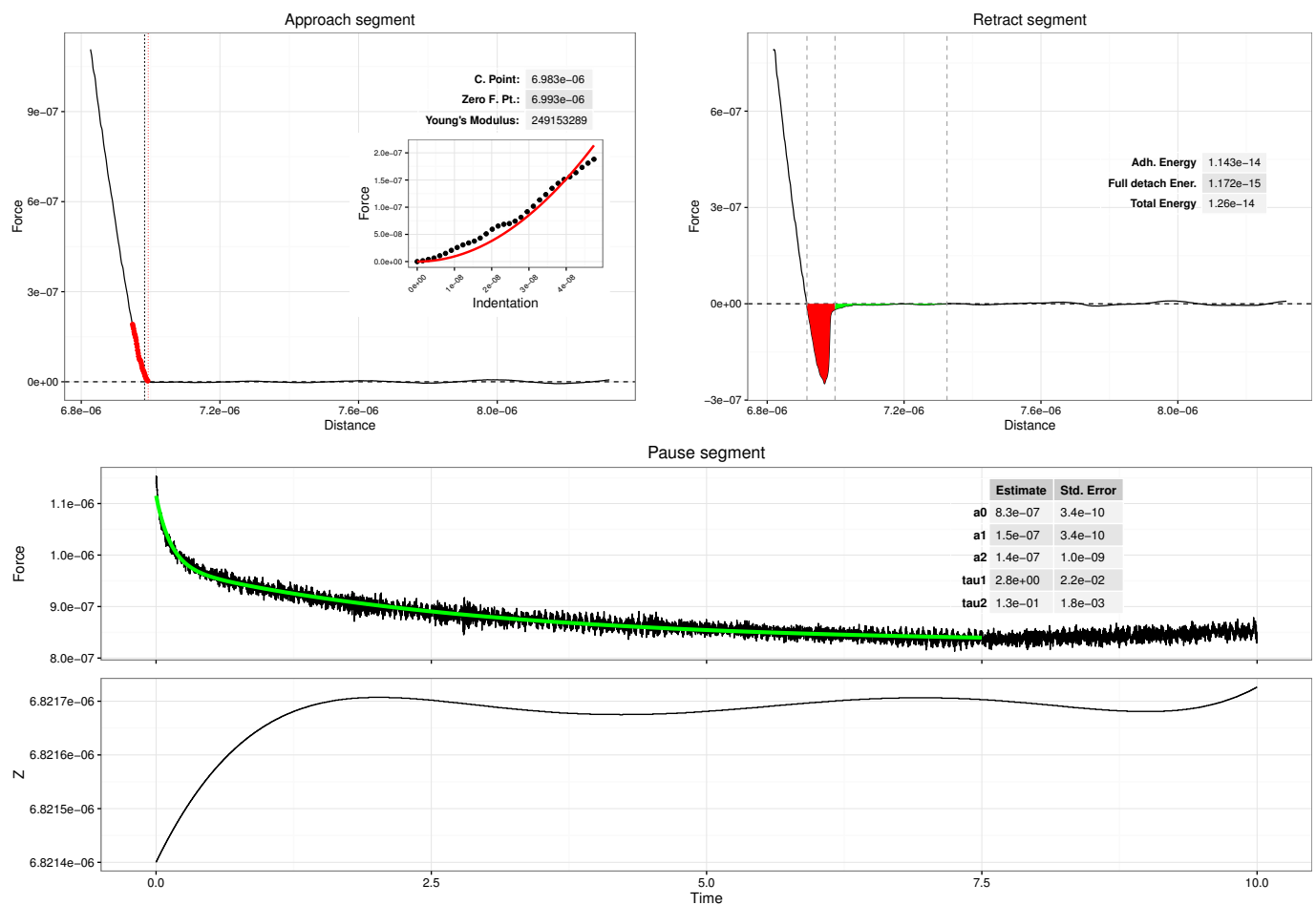

Figure 10: Summary plots. Information and plots given by the summary function.

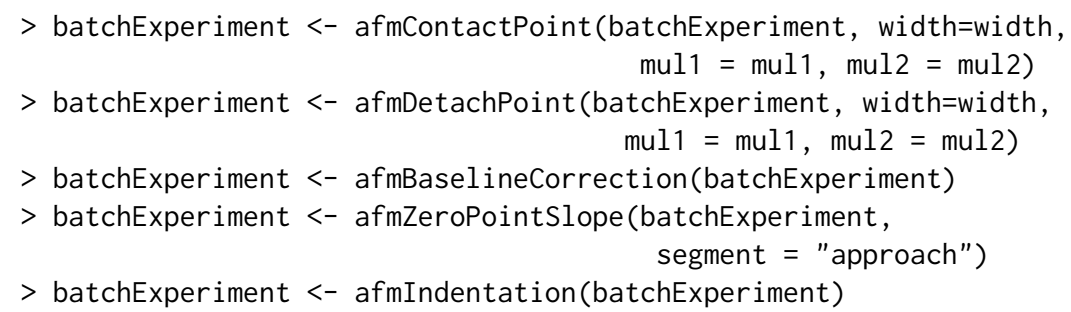

\section{Curve analysis.}

$>$ batchExperiment <- afmYoungModulus(batchExperiment, thickness $=2.5 \mathrm{e}-7$, geometry = "paraboloid", params $=\operatorname{list}(\mathrm{R}=1 \mathrm{e}-8)$ )

$>$ batchExperiment $<-$ afmExpDecay (batchExperiment, nexp $=2$, type $=$ "CH", plt $=$ FALSE, tmax $=5$ )

$>$ batchExperiment <- afmAdhesionEnergy (batchExperiment, width $=5, \mathrm{mul}=15$ )

After some warning messages due to the afmExpDecay function telling us that we did not provide some initial values for the Levenberg-Mardquart algorithm, we will find that now our "afmexperiment" structure is a list of 14 "afmdata" class variables, each one with 8 fields:

$>\operatorname{head}($ summary (batchExperiment))

force-save-2016.07.27-16.56.36.140.txt 8 Length Class Mode force-save-2016.07.27-16.57.00.672.txt 8 afmdata list force-save-2016.07.27-16.57.25.194.txt 8 afmdata list ...

3. Extracting the results: Once all the analyses are performed, we need to extract the information from the "afmexperiment" list and store it in spreadsheet-like data frames, since they are more suitable formats for further analysis or plotting.

To that aim we use the afmExtract function.

$>$ parameters <- afmExtract(batchExperiment,

$$
\begin{aligned}
& \text { params = list("YM", "AE", "ED"), } \\
& \text { opt.param = "type") }
\end{aligned}
$$


The parameter params is a list with the parameters we want to extract (YM stands for "Young's Modulus", AE for "Adhesion Energies" and ED for "Exponential decay"). The parameter opt.param is an optional parameter with additional information that we may want to store in our data frames (like factors describing the experiments). In this case we set 'opt. param = "type"' so we include the information relative to the type of experiment ("PAH" or "CHI").

The result of the afmExtract function is a list with two data frames. The first one storing the Young's Modulus and the Adhesion Energies, and the second one containing the exponential decay results (coefficients and standard errors).

4. Plotting the results. Plotting the parameters is now straightforward, provided the dplyr package for data frame manipulation is installed and loaded:

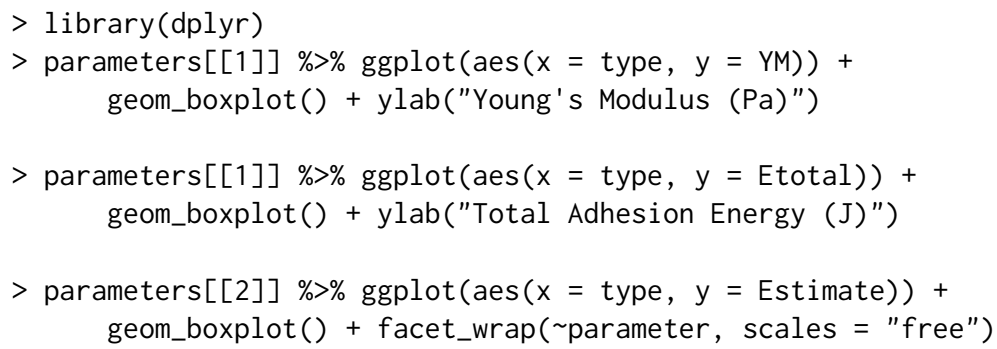

Young's Modulus results are depicted in Figure 11 (left plot), total Adhesion Energy results in Figure 11 (right plot), and the different parameter estimates for the exponential decays are shown in Figure 12.
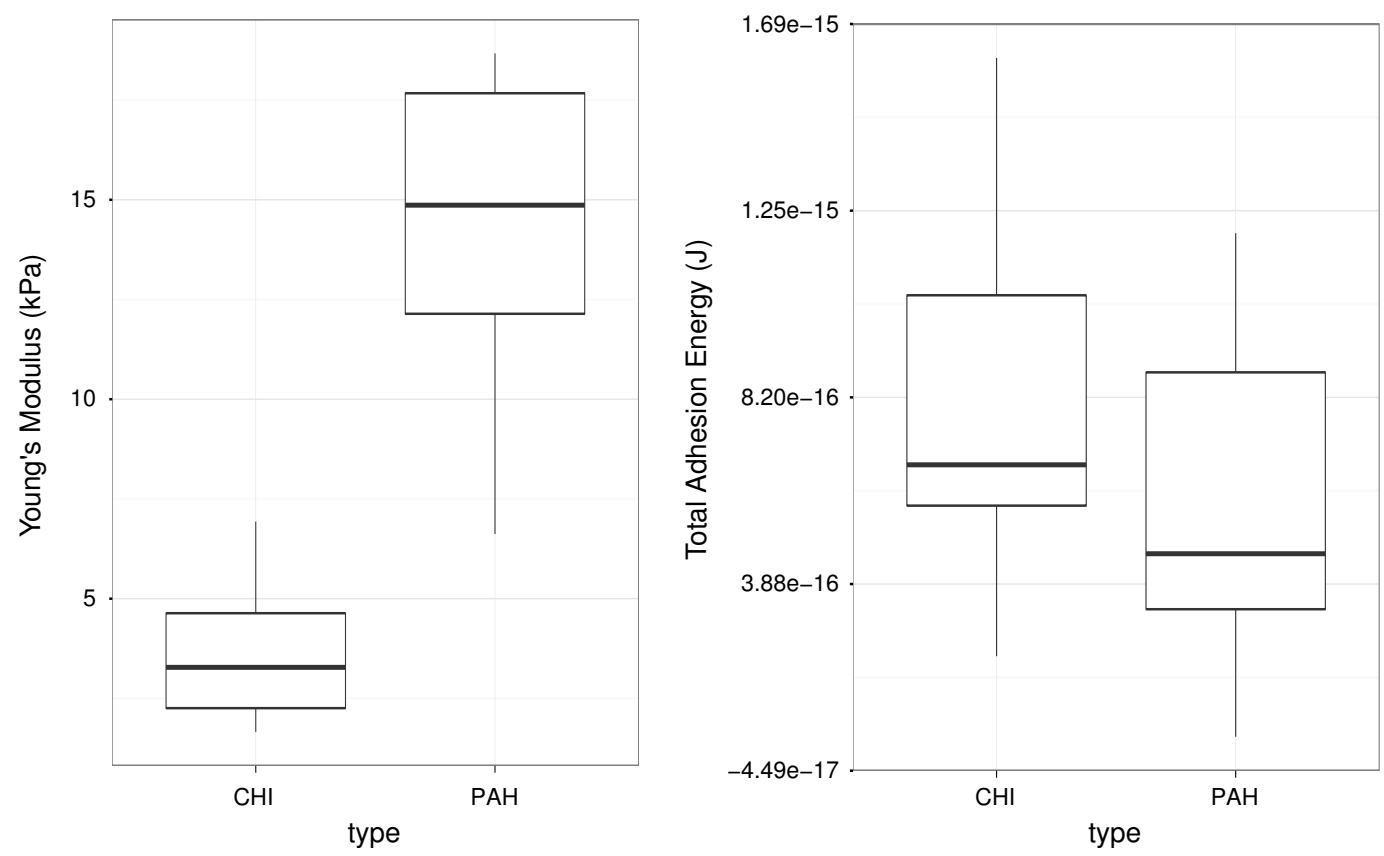

Figure 11: Batch processing: Left: Young's Modulus vs. Experiment type boxplot. Right: "Total Adhesion Energy" vs. "Experiment type" boxplot. Both plots extracted from an "afmexperiment" data class variable.

\section{Conclusions}

The R programming language has became in the recent years the "lingua franca" of statistics and data science. The number of users contributed packages has increased exponentially reaching more 10000 packages. However, although there are several packages for AFM image processing at CRAN, up to our knowledge, this is the first $\mathrm{R}$ package for force spectroscopy analysis.

Our goal has been to create a set of functions for the automatic analysis of force-distance curves while being flexible enough to be easily extended by adding algorithms that allow easier analysis in more and more different types of AFM experiments. 


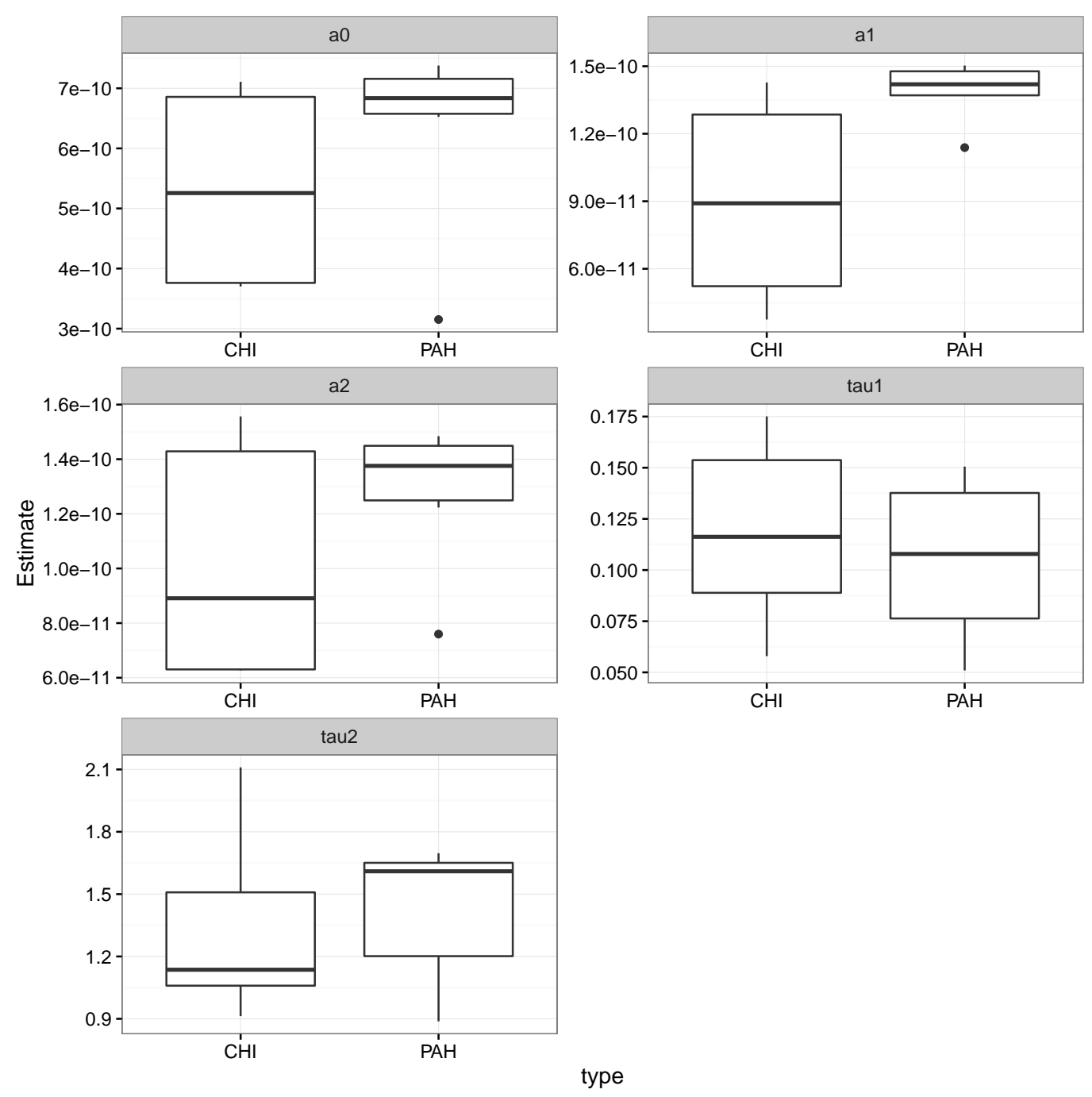

Figure 12: Batch processing: Exponential decay. Boxplot of the values of the estimated parameters in the double exponential decay fit for each experiment type.

For example, in the scope of event detection, the use of wavelets has proven to be very effective in determining protein folding events (García-Massó et al., 2016) or in the detection of jumps in the retract segment of the curve, that may be associated with the formation of tethers (Benítez and Bolós, 2017). Thus, new directions in the development of this afmToolkit could be the inclusion of peak detectors.

We sincerely hope that this package will be found useful by the force - spectroscopy scientists and we are willing to recieve feedback from the users.

\section{Acknowledgements}

The authors would like to thank Julia Miholich for the testing and bug-finding and Alberto Cencerrado, Jagoba Iturri and Xavi García for making the measurements.

\section{Bibliography}

J. Alcaraz, L. Buscemi, M. Grabulosa, X. Trepat, B. Fabry, R. Farré, and D. Navajas. Microrheology of human lung epithelial cells measured by atomic force microscopy. Biophysical Journal, 84(3): 2071-2079, 2003. URL https://doi.org/10.1016/S0006-3495(03)75014-0. [p291]

B. Andreopoulos and D. Labudde. Efficient unfolding pattern recognition in single molecule force 
spectroscopy data. Algorithms for Molecular Biology, 6(1), 2011. URL https://doi .org/10.1186/ 1748-7188-6-16. [p291]

B. Auguie. gridExtra: Miscellaneous Functions for "Grid" Graphics, 2016. URL https: //CRAN. R-project. org/package=gridExtra. R package version 2.2.1. [p292]

R. Benítez and V. Bolós. Searching events in afm force-extension curves: A wavelet approach. Microscopy research and technique, 80(1):153-159, 2017. URL https://doi .org/10.1002/jemt. 22720. [p305]

R. Benitez and J. Toca-Herrera. Looking at cell mechanics with atomic force microscopy: experiment and theory. Microscopy research and technique, 77(11):947-958, 2014. URL https://doi .org/10.1002/ jemt. 22419. [p291]

R. Benítez, S. Moreno-flores, V. J. Bolós, and J. L. Toca-Herrera. A new automatic contact point detection algorithm for afm force curves. Microscopy research and technique, 76(8):870-876, 2013. URL https://doi.org/10.1002/jemt.22241. [p291,295]

R. Best, D. Brockwell, J. Toca-Herrera, A. Blake, D. Smith, S. Radford, and J. Clarke. Force mode atomic force microscopy as a tool for protein folding studies. Analytica Chimica Acta, 479(1):87-105, 2003. URL https://doi .org/10.1016/S0003-2670(02)01572-6. [p291]

M. Borkovec, I. Szilagyi, I. Popa, M. Finessi, P. Sinha, P. Maroni, and G. Papastavrou. Investigating forces between charged particles in the presence of oppositely charged polyelectrolytes with the multi-particle colloidal probe technique. Advances in colloid and interface science, 179-182:85-98, nov 2012. URL https://doi.org/10.1016/j.cis.2012.06.005. [p291]

H.-J. Butt, B. Cappella, and M. Kappl. Force measurements with the atomic force microscope: Technique, interpretation and applications. Surface Science Reports, 59(1-6):1-152, oct 2005. URL https://doi.org/10.1016/j. surfrep.2005.08.003. [p291]

S. L. Crick and F. C.-P. Yin. Assessing micromechanical properties of cells with atomic force microscopy: importance of the contact point. Biomechanics and modeling in mechanobiology, 6(3):199-210, apr 2007. URL https://doi.org/10.1007/s10237-006-0046-x. [p291]

W. A. Ducker, T. J. Senden, and R. M. Pashley. Direct measurement of colloidal forces using an atomic force microscope. Nature, 353(6341):239-241, 1991. URL https://doi .org/10.1038/353239a0. [p291]

T. V. Elzhov, K. M. Mullen, A.-N. Spiess, and B. Bolker. minpack.lm: R Interface to the LevenbergMarquardt Nonlinear Least-Squares Algorithm Found in MINPACK, Plus Support for Bounds, 2015. URL https: //CRAN.R-project.org/package=minpack. Im. R package version 1.2-0. [p292, 299]

S. Garcia-Manyes and F. Sanz. Nanomechanics of lipid bilayers by force spectroscopy with AFM: a perspective. Biochimica et biophysica acta, 1798(4):741-9, apr 2010. URL https://doi .org/10.1016/ j. bbamem. 2009.12.019. [p291]

X. García-Massó, M. C. Huber, J. Friedmann, L. M. Gonzalez, S. M. Schiller, and J. L. Toca-Herrera. Automated detection of protein unfolding events in atomic force microscopy force curves. Microscopy Research and Technique, 79(11):1105-1111, 2016. URL https://doi .org/10.1002/jemt.22764. [p305]

N. Gavara. Combined strategies for optimal detection of the contact point in AFM force-indentation curves obtained on thin samples and adherent cells. Scientific Reports, 6:21267, Feb. 2016. URL https://doi.org/10.1038/srep21267. [p295]

H. G. Hansma, I. Revenko, K. Kim, and D. E. Laney. Atomic Force Microscopy of Long and Short Double-Stranded, Single-Stranded and Triple-Stranded Nucleic Acids. Nucleic Acids Research, 24(4): 713-720, feb 1996. URL https://doi .org/10.1093/nar/24.4.713. [p291]

P. Hinterdorfer, W. Baumgartner, H. J. Gruber, K. Schilcher, and H. Schindler. Detection and localization of individual antibody-antigen recognition events by atomic force microscopy. Proceedings of the National Academy of Sciences, 93(8):3477-3481, apr 1996. URL https://10.1073/pnas.93.8.3477. [p291]

B. Kainz, E. A. Oprzeska-Zingrebe, and J. L. Toca-Herrera. Biomaterial and cellular properties as examined through atomic force microscopy, fluorescence optical microscopies and spectroscopic techniques. Biotechnology journal, 9(1):51-60, jan 2014. URL https://doi .org/10.1002/biot. 201300087. [p291] 
S. Kasas and G. Dietler. Probing nanomechanical properties from biomolecules to living cells. Pflïgers Archiv : European journal of physiology, 456(1):13-27, apr 2008. URL https://doi.org/10.1007/ s00424-008-0448-y. [p291]

S. Kasas, B. M. Riederer, S. Catsicas, B. Cappella, and G. Dietler. Fuzzy logic algorithm to extract specific interaction forces from atomic force microscopy data. Review of Scientific Instruments, 71(5): 2082-2086, 2000. URL https://doi.org/10.1063/1.1150583. [p291]

Y. G. Kuznetsov, J. J. Dowell, J. A. Gavira, J. D. Ng, and A. McPherson. Biophysical and atomic force microscopy characterization of the RNA from satellite tobacco mosaic virus. Nucleic acids research, 38(22):8284-94, dec 2010. URL https://doi.org/10.1093/nar/gkq662. [p291]

D. C. Lin, E. K. Dimitriadis, and F. Horkay. Robust strategies for automated AFM force curve analysis-I. Non-adhesive indentation of soft, inhomogeneous materials. Journal of biomechanical engineering, 129 (3):430-40, jun 2007a. URL https://doi .org/10.1115/1.2720924. [p291, 295]

D. C. Lin, E. K. Dimitriadis, and F. Horkay. Robust strategies for automated AFM force curve analysisII: adhesion-influenced indentation of soft, elastic materials. Journal of biomechanical engineering, 129 (6):904-12, dec 2007b. URL https://doi .org/10.1115/1.2800826. [p295]

A. E. Lopez, S. Moreno-Flores, D. Pum, U. B. Sleytr, and J. L. Toca-Herrera. Surface dependence of protein nanocrystal formation. Small, 6(3):396-403, 2010. URL https://doi .org/10.1002/smll. 200901169. [p291]

P. E. Marszalek, H. Lu, H. Li, M. Carrion-Vazquez, A. F. Oberhauser, K. Schulten, and J. M. Fernandez. Mechanical unfolding intermediates in titin modules. Nature, 402(6757):100-3, nov 1999. URL https://doi.org/10.1038/47083. [p291]

K. A. Melzak and J. L. Toca-Herrera. Atomic force microscopy and cells: Indentation profiles around the afm tip, cell shape changes, and other examples of experimental factors affecting modeling. Microscopy Research and Technique, 78(7):626-632, 2015. URL https://doi . org/10. 1002/ jemt. 22522. [p291]

S. Moreno-Flores and J. L. Toca-Herrera. Hybridizing Surface Probe Microscopies: Toward a Full Description of the Meso- and Nanoworlds, volume 8. CRC. Taylor and Francis, Boca Raton, 2013. [p291]

S. Moreno-Flores, R. Benitez, M. dM Vivanco, and J. L. Toca-Herrera. Stress relaxation and creep on living cells with the atomic force microscope: a means to calculate elastic moduli and viscosities of cell components. Nanotechnology, 21(44):445101, nov 2010a. URL https://doi . org/10. 1088/09574484/21/44/445101. [p298]

S. Moreno-Flores, R. Benitez, M. D. Vivanco, and J. L. Toca-Herrera. Stress relaxation microscopy: imaging local stress in cells. Journal of biomechanics, 43(2):349-54, jan 2010b. URL https: //doi . org/ 10.1016/j.jbiomech.2009.07.037. [p298]

D. J. Müller and Y. F. Dufrêne. Atomic force microscopy as a multifunctional molecular toolbox in nanobiotechnology. Nature nanotechnology, 3(5):261-9, may 2008. URL https: //doi . org/10. 1038/ nnano. 2008.100. [p291]

J. L. Ortega-Vinuesa, P. Tengvall, and I. Lundström. Molecular packing of HSA, IgG, and fibrinogen adsorbed on silicon by AFM imaging. Thin Solid Films, 324(1-2):257-273, 1998. URL https://doi . org/10.1016/50040-6090(98)00363-0. [p291]

E. Riande, R. Díaz-Calleja, M. G. Prolongo, R. M. Masesgosa, and C. Salom. Polymer viscoelasticity : stress and strain in practice. CRC Press, New York, 1999. [p298]

M. Rief. Single Molecule Force Spectroscopy on Polysaccharides by Atomic Force Microscopy. Science, 275(5304):1295-1297, feb 1997. URL https: //doi .org/10.1126/science.275.5304.1295. [p291]

D. Rudoy, S. G. Yuen, R. D. Howe, and P. J. Wolfe. Bayesian change-point analysis for atomic force microscopy and soft material indentation. Journal of the Royal Statistical Society. Series C: Applied Statistics, 59(4):573-593, 2010. URL https://doi.org/10.1111/j.1467-9876.2010.00715.x. [p295]

H. Wickham. ggplot2: Elegant Graphics for Data Analysis. Springer, New York, 2016a. [p292]

H. Wickham. scales: Scale Functions for Visualization, 2016b. URL https://CRAN. R-project.org/ package=scales. R package version 0.4.0. [p292]

H. Wickham and W. Chang. devtools: Tools to Make Developing R Packages Easier, 2016. URL https: //CRAN.R-project. org/package=devtools. R package version 1.12.0. [p292] 
H. Wickham and R. Francois. dplyr: A Grammar of Data Manipulation, 2015. URL https://CRAN. Rproject. org/package=dplyr. R package version 0.4.3. [p292]

Rafael Benítez

Department of Bussiness Mathematics. Univeristy of Valencia Avda. Tarongers s/n. 46022. Valencia Spain

rabesua@uv.es

Vicente J. Bolós

Department of Bussiness Mathematics. Univeristy of Valencia Avda. Tarongers s/n. 46022. Valencia

Spain

vicente.bolos@uv.es

José-Luis Toca-Herrera

Department of Nanobiotechnology. Institute for Biophysics.

University of Natural Resources and Life Sciences - BOKU Wien

Simon Zeisel Haus. Muthgasse 11/II.1190 Wien

Austria

jose. toca-herrera@boku.ac.at 